\title{
Is Behçet's syndrome associated with an increased risk of ischemic heart disease? A real-world evidence in Taiwan
}

\author{
Chun-Yu Lin ${ }^{1 *} \mathbb{D}$, Hung-An Chen ${ }^{2,3}$, Chun-Hsin Wu ${ }^{4}$, Yu-Jih Su ${ }^{5,6}$, Tsai-Ching Hsu ${ }^{7}$ and Chung-Yuan Hsu ${ }^{8^{*}}$
}

\begin{abstract}
Background: A variety of chronic inflammatory diseases are linked to ischemic heart disease (IHD); however, this association is less well studied in patients with Behçet's syndrome (BS). The primary objective of this study was to examine the impact of $\mathrm{BS}$ on the risk of $\mathrm{IHD}$. The secondary objective was to estimate the long-term mortality risk in patients with BS.
\end{abstract}

Methods: Using a retrospective cohort design based on the Taiwan National Health Insurance Database, patients diagnosed with BS between 2000 and 2013, without prior history of IHD, were compared to non-BS individuals. The BS and non-BS cohorts were matched with a 1:2 ratio by propensity score, accounting for the following confounders: age, sex, year of index date, comorbidities, and drug exposure. Cox proportional hazard regression was used to derive the hazard ratio (HR) for IHD and mortality. The long-term survival rate was estimated using the Kaplan-Meier method.

Results: After propensity score matching, a total of 1554 patients newly diagnosed with BS and 3108 control subjects were identified. The incidence rate of IHD in the BS and control groups was 2.7 and 2.9 per 1000 person-years, respectively. The risk of IHD was comparable between BS and control cohorts [adjusted HR, 1.03; 95\% confidence interval (Cl), 0.66 to 1.62]. The 5- and 10-year survival rate of BS patients was $96.8 \%$ and $95.0 \%$, respectively. Patients with BS exhibited a significantly higher risk of mortality than the sex- and agematched general population (adjusted HR, 1.73; 95\% Cl, 1.30 to 2.32).

Conclusion: Unlike other chronic systemic autoimmune disorders, BS does not appear to be associated with an excess risk of $\mathrm{HD}$.

Keywords: Behçet's syndrome, Ischemic heart disease, Long-term mortality

\footnotetext{
*Correspondence: linchunyumed@gmail.com; chungyuango@gmail.com

'Division of Rheumatology, Department of Internal Medicine, National Cheng

Kung University Hospital, College of Medicine, National Cheng Kung

University, No.138, Sheng Li Road, Tainan 704, Taiwan

${ }^{8}$ Division of Rheumatology, Allergy and Immunology, Department of Internal Medicine, Kaohsiung Chang Gung Memorial Hospital, Chang Gung University

College of Medicine, No. 123, Ta Pei Road, Niao Sung District, Kaohsiung

83301, Taiwan

Full list of author information is available at the end of the article
}

(c) The Author(s). 2021 Open Access This article is licensed under a Creative Commons Attribution 4.0 International License, which permits use, sharing, adaptation, distribution and reproduction in any medium or format, as long as you give appropriate credit to the original author(s) and the source, provide a link to the Creative Commons licence, and indicate if changes were made. The images or other third party material in this article are included in the article's Creative Commons licence, unless indicated otherwise in a credit line to the material. If material is not included in the article's Creative Commons licence and your intended use is not permitted by statutory regulation or exceeds the permitted use, you will need to obtain permission directly from the copyright holder. To view a copy of this licence, visit http://creativecommons.org/licenses/by/4.0/ The Creative Commons Public Domain Dedication waiver (http://creativecommons.org/publicdomain/zero/1.0/) applies to the data made available in this article, unless otherwise stated in a credit line to the data. 


\section{Introduction}

Behçet's syndrome (BS) is a multi-systemic autoinflammatory disorder characterized by recurrent oral and/or genital ulcerations, uvea inflammation, and vasculitis [1-3]. Epidemiological studies have shown that the incidence and prevalence of BS varies across countries and geographic areas, being highest in the Mediterranean countries and the Far East area [2-4]. The clinical features and expressions of BS also exhibit geographic variation. For example, the involvement of the gastrointestinal tract is more frequent among patients with BS in Asia than in Turkey $[5,6]$. Regarding the survival of patients with BS, some studies have been performed in the last century, mostly in Turkey [7]. Recently, the mortality risk for patients with BS was reported by a study in the United Kingdom (UK) [8]. Despite the geographical variation in the clinical presentation of BS, it is uncertain whether the long-term prognosis of BS also differs across different ethnic populations. Moreover, few studies have addressed the long-term mortality risk of BS in the Chinese population at a general population level.

A number of chronic inflammatory disorders, such as rheumatoid arthritis (RA), systemic lupus erythematosus (SLE), and idiopathic inflammatory myopathy, have been strongly linked with atherosclerosis and ischemic heart disease (IHD) in recent decades [9-11]. Systemic inflammation has been recognized as a key player in the initiation and progression of atherosclerotic heart disease in patients with chronic autoimmune diseases [10]. Although major vascular involvement is far more common in the disease course of BS, arterial lesions usually manifest as pulmonary arterial aneurysms, whereas coronary artery disease has not been recognized as a prominent feature of BS in earlier clinical studies [12, 13]. Moreover, a study assessing subclinical atherosclerosis in the carotid and femoral arteries has shown that the frequency of atherosclerotic plaques is comparable between patients with BS and healthy control subjects [14]. Another study using computed tomography to investigate the condition of the coronary artery found a relatively low frequency of coronary artery calcification in male patients with BS, even in those with diffuse large vessel disease [15]. Therefore, whether the risk of IHD is as high in BS as in other chronic inflammatory diseases remains unclear.

We conducted this large-scale cohort study to determine whether or not the risk of clinically significant IHD was elevated in patients with $\mathrm{BS}$ compared to that in the non-BS population. We also sought to determine the long-term survival rate of BS patients and compare their mortality risk with that of matched individuals without BS.

\section{Methods}

\section{Study design and data source}

This retrospective follow-up study was conducted using the National Health Insurance Research Database (NHIR
D) in Taiwan. A mandatory National Health Insurance (NHI) program was launched in Taiwan in 1995, and over $99 \%$ of the population had been enrolled in this program until 2013. The NHI program provides broad health care coverage, including ambulatory care, inpatient care, dental care, prescription drugs, and surgical procedures, for Taiwan's residents. Thus, NHIRD, which was constructed based on the NHI program and contains detailed health care data, is one of the largest databases worldwide and is used extensively for epidemiological and long-term follow-up studies [16, 17]. The identification number of patients in NHIRD is encrypted to ensure privacy protection. Therefore, informed consent in our study was waived, and the National Cheng Kung University Hospital Institutional Review Board approved the study protocol (A-EX-109-017). The study flow chart of the selection process of patients and comparison cohort is summarized in Fig. 1.

\section{Study cohort}

Adult patients (aged > 20 years) with BS were identified during the period from January 1, 2000, to December 31, 2013, using the International Classification of Diseases, Ninth Revision, Clinical Modification (ICD-9-CM) code 136.1, from outpatient or inpatient records in the NHIR D. To ensure the accuracy of the diagnosis, patients who were considered to have BS were also required to hold a catastrophic illness certificate for BS, in addition to having corresponding diagnostic codes. Patients with severe disorders, such as cancer, autoimmune diseases, endstage kidney disease requiring dialysis, or type 1 diabetes, can apply for a catastrophic illness certificate under the Taiwan NHI system. Patients who were issued such a certificate may be exempted from copayment when seeking medical care related to such illness. To obtain this certificate for BS, medical records, laboratory results, and image findings are reviewed by two boardcertified rheumatologists, and the International Study Group (ISG) criteria for BS should be fulfilled [18]. Patients without a certificate were excluded from our study, ensuring proper identification of BS.

The index date was defined as the date of first diagnosis of BS. We also screened the 2-year period before the index date to ensure that no diagnosis of BS had been made during that period, and we only included incident cases of BS in our study. Patients who developed IHD before the index date were also excluded from the study.

\section{Comparison (control) group}

Patients without BS were selected from the subset of NHIRD identified between 2000 and 2013. The index dates for the non-BS cohort were randomly assigned, corresponding to the distribution of those in the BS cohort. The exclusion criteria for the BS cohort were also 


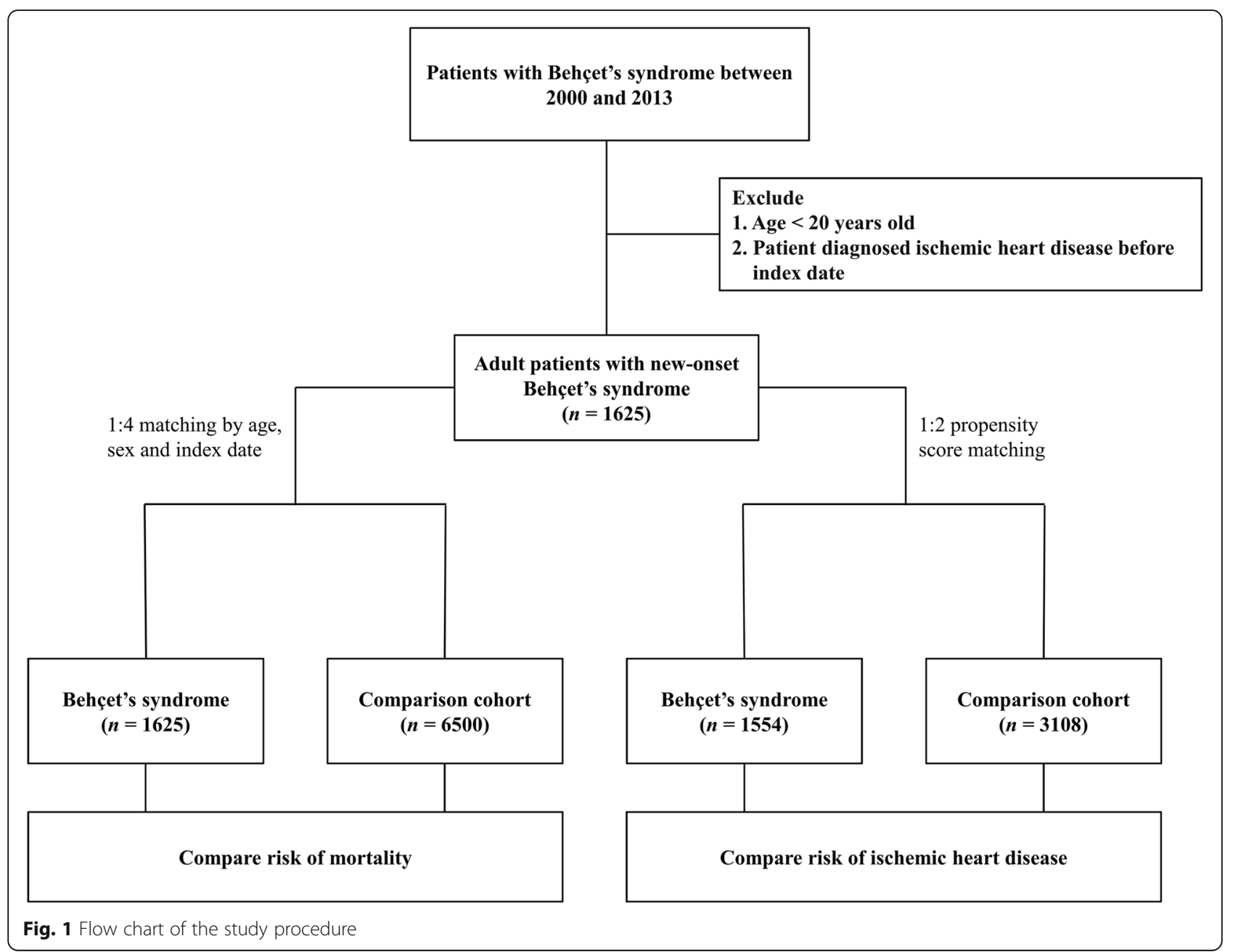

applied to the non-BS cohort; i.e., patients aged $<20$ years or experiencing IHD before the index date were not included in the control group.

\section{Assessment of covariates}

Demographic information, including age and sex, was retrieved from the database. Comorbid diseases were searched in the 2-year period before the index date. Patients were designated as having a specific comorbidity if they had received corresponding diagnostic codes at least one time in the inpatient claims or at least three times in the outpatient claims. Comorbidities analyzed in the present study were as follows: diabetes (ICD-9CM code 250), hypertension (ICD-9-CM codes 401405), dyslipidemia (ICD-9-CM code 272), chronic obstructive lung disease (COPD; ICD-9-CM codes 491, 492, 496), and chronic kidney disease (ICD-9-CM codes 580-588). Exposure to various medications, including non-steroidal anti-inflammatory drugs (NSAIDs), systemic corticosteroids, statins, and aspirin, was also recorded within the 90-day period of the index date.

\section{Study outcomes}

The main outcome of this study was the occurrence of IHD. Development of IHD was ascertained by ICD-9CM codes 410 (acute myocardial infarction) and 411 (acute and subacute forms of ischemic heart disease) in the principal diagnosis of inpatient claims. The accuracy of the diagnosis of acute myocardial infarction was validated in Taiwan's NHIRD with a positive predictive value of 0.93 when using only the principal diagnosis [19]. When analyzing the main outcome, patients were followed up until the development of IHD, death, or the end of the study (December 31, 2013), whichever occurred first. The secondary outcome was mortality. When analyzing the secondary outcome, patients were followed up until death or the end of the study (December 31, 2013), whichever occurred first.

\section{Process of propensity score matching in the assessment of IHD}

We carried out propensity score matching for analyzing the risk of IHD in the BS versus non-BS group, in order 
to reduce the confounding effects of age, sex, comorbidities, and medication use on the outcome. We constructed a logistic regression model with patients having BS or not as the dependent variable and age, sex, year of index date, diabetes, hypertension, COPD, hyperlipidemia, use of stains, aspirin, corticosteroids, and NSAIDs as independent variables, in order to estimate the propensity score. Then, each patient with BS was matched to two subjects without BS using a caliper width of 0.2 of the pooled standard deviation of the logit of the propensity score. The quality of balance of covariates between BS and non-BS groups was checked by the mean standardized difference. If this difference was less than 0.1 , the balance of confounding variables was considered to be acceptable [20].

\section{Matching for age, sex, and year of index date in the assessment of mortality}

For estimating the mortality risk in patients with BS compared with that in the general population, we constructed another comparison control cohort, which was matched with the BS cohort with a 1:4 ratio (Fig. 1). The matching variables in this analysis included age, sex, and year of index date.

\section{Power and statistical analysis}

The study design assumed an IHD event rate of $3 \%$ in the non-BS group at 10 years' follow-up, and a hazard ratio (HR) of 2.0 in the BS group versus non-BS group. Then, the sample size was calculated with the use of a two-sided test at the 0.05 significance level and the $90 \%$ power level. It was determined that 827 patients with BS and 1652 controls should be included in the current study.

Continuous and categorical variables were presented as mean \pm standard deviation and percentage, respectively. The Kaplan-Meier method was used to calculate the cumulative incidence of IHD and the survival rate in the BS and matched control cohorts. A log-rank test was used to determine whether a significant difference existed with respect to the incidence of IHD and survival between BS and non-BS groups. The doubly robust method, combining the regression model for outcomes and propensity score models, was used to derive the final effect estimates of BS on the IHD incidence [21, 22]. The advantage of this method was that the estimates were unbiased as long as either model (propensity score or outcome regression) was correctly specified. Specifically, we used a Cox proportional hazard regression model adjusting for sex, age, comorbidities, and medications to derive the impact of BS on IHD occurrence after propensity score matching of the BS and control groups. We further performed a sensitivity analysis using a Fine and Gray competing risk regression model [23], considering the event of death as a competing event when analyzing the primary outcome of IHD occurrence. The threshold of the significance level was set at a two-sided $P$ value $<0.05$. All data management, graph preparation, and statistical analyses were performed using Stata version 13 software (StataCorp, College Station, TX, USA).

\section{Results}

A total of 1625 patients with BS were identified in the period from 2000 to 2013 . Table 1 presents the demographic information, baseline characteristics, and medication exposure in the BS and control cohorts. After propensity score matching, 1554 patients with BS and 3108 matched control subjects remained for further analysis in our study. The mean standardized difference of all variables between the BS and control groups after matching was less than 0.1. Female sex accounted for $58 \%$ of the BS cohort. The mean age of patients with BS was $39.2 \pm 12.0$ years. The most prevalent comorbid disease in patients was hypertension, followed by hyperlipidemia, diabetes, chronic kidney disease, and COPD. However, the percentage of these comorbidities was less than $10 \%$ in all cases. One third of patients with BS had received corticosteroid treatment, while statin and aspirin were less prescribed.

\section{Incidence and risk of IHD}

Among the 1554 patients with BS and 3108 matched control subjects, $29(1.9 \%)$ and $63(2.0 \%)$, respectively, developed IHD during follow-up (Table 2). The incidence rate of IHD in BS and control cohorts was 2.7 and 2.9 per 1000 person-years, respectively. Figure 2 presents the cumulative incidence of IHD; no significant difference was detected between groups (log-rank test, $\mathrm{P}$ value $=0.68$ ). Cox regression analysis, adjusting for age, sex, comorbidities, and drug use, revealed that the risk of IHD was similar between patients and control subjects (HR, 1.03; 95\% confidence interval [CI], 0.66 to 1.62). Sensitivity analysis using a competing risk regression model also showed similar results (sub-distribution HR, 1.00 ; $95 \%$ CI, 0.64 to 1.57 ).

\section{Mortality rate}

A total of 1625 patients with BS and 6500 control subjects were included in the analysis of mortality risk during the study period (Table 3). Among them, 65 (4.0\%) and $157(2.4 \%)$ subjects in the BS and control cohort, respectively, died. The rate of mortality in patients and controls was 5.60 and 3.33 per 1000 person-years, respectively. The 3-, 5-, and 10-year survival rates of patients were $98.2 \%, 96.8 \%$, and $95.0 \%$, respectively, while the respective rates in control subjects were 99.1\%, 98.5\%, and $96.8 \%$ (Fig. 3A). The log-rank test indicated that patients exhibited a higher mortality risk compared 
Table 1 Summary of demographics and characteristics of patients with Behçet's syndrome and controls without Behçet's syndrome

\begin{tabular}{|c|c|c|c|c|}
\hline \multirow[t]{2}{*}{ Characteristics } & \multirow{2}{*}{$\begin{array}{l}\text { Before propensity score matching } \\
\text { Behçet's syndrome } \\
(\mathrm{n}=1625)\end{array}$} & \multicolumn{3}{|c|}{ After propensity score matching } \\
\hline & & $\begin{array}{l}\text { Behçet's syndrome } \\
(\mathrm{n}=1554)\end{array}$ & $\begin{array}{l}\text { Control } \\
(n=3108)\end{array}$ & $\overline{\text { Standardized mean difference }}$ \\
\hline Sex, n (\%) & & & & 0.04 \\
\hline Female & $942(58.0)$ & $901(58.0)$ & $1735(56.0)$ & \\
\hline Male & $683(42.0)$ & $653(42.0)$ & $1373(44.0)$ & \\
\hline Age group, $n(\%)$, years & & & & 0.001 \\
\hline $18-44$ & $1131(69.6)$ & $1064(68.5)$ & $2130(68.5)$ & \\
\hline$\geqq 45$ & $494(30.4)$ & $490(31.5)$ & $978(31.5)$ & \\
\hline Age, mean $\pm S D$, years & $38.8 \pm 11.9$ & $39.2 \pm 12.0$ & $39.1 \pm 12.2$ & 0.008 \\
\hline \multicolumn{5}{|l|}{ Comorbidities, n (\%) } \\
\hline Diabetes & $39(2.4)$ & $39(2.5)$ & $88(2.8)$ & -0.020 \\
\hline Hypertension & $104(6.4)$ & $103(6.6)$ & $211(6.7)$ & -0.006 \\
\hline Hyperlipidemia & $40(2.5)$ & $40(2.6)$ & $98(3.1)$ & -0.035 \\
\hline Chronic kidney disease & $21(1.3)$ & $21(1.4)$ & $41(1.3)$ & 0.003 \\
\hline COPD & $21(1.3)$ & $21(1.4)$ & $40(1.3)$ & 0.006 \\
\hline \multicolumn{5}{|l|}{ Medications, n (\%) } \\
\hline NSAID & $465(28.6)$ & $405(26.1)$ & $802(25.8)$ & 0.006 \\
\hline Corticosteroid & $589(36.3)$ & $520(33.5)$ & 1048 (33.7) & -0.005 \\
\hline Statin & $16(1.0)$ & $16(1.0)$ & $46(1.4)$ & -0.04 \\
\hline Aspirin & $37(2.3)$ & $33(2.1)$ & $67(2.2)$ & -0.002 \\
\hline
\end{tabular}

$C O P D$, chronic obstructive pulmonary disease; NSAID, nonsteroidal anti-inflammatory drug; $S D$, standard deviation

with control patients ( $\mathrm{P}$ value $<0.001)$. Cox regression model analysis showed that the risk of death in the BS group was $73 \%$ higher than that in the sex- and agematched control group (HR, 1.73; 95\% CI, 1.30 to 2.32). In addition, the risk of death was higher for male than for female patients with BS (P value = 0.04) (Fig. 3B).

\section{Discussion}

We conducted a retrospective study to assess the risk of IHD and mortality in a representative sample of patients

Table 2 Incidence rate and relative risk for ischemic heart disease in patients with Behçet's syndrome and in propensity score-matched control subjects

\begin{tabular}{lll}
\hline & $\begin{array}{l}\text { Behçet's syndrome } \\
(\mathbf{n}=\mathbf{1 5 5 4})\end{array}$ & $\begin{array}{l}\text { Control } \\
\mathbf{( n = 3 1 0 8 )}\end{array}$ \\
\hline $\begin{array}{l}\text { Ischemic heart disease } \\
\text { Cases, n (\%) }\end{array}$ & $29(1.9)$ & $63(2.0)$ \\
Person-years & 10,909 & 21,554 \\
IR $(95 \% \mathrm{Cl})^{*}$ & $2.7(1.9-3.8)$ & $2.9(2.2-3.7)$ \\
aHR $(95 \% \mathrm{Cl})^{* *}$ & $1.03(0.66-1.62)$ & reference \\
SHR & $1.00(0.64-1.57)$ & reference \\
\hline
\end{tabular}

"Expressed per 1000 person-years

"*Adjusted for age, sex, diabetes, hypertension, chronic kidney disease, COPD, and use of NSAIDs, corticosteroids, statins, and aspirin

COPD, chronic obstructive pulmonary disease; NSAID, nonsteroidal antiinflammatory drug; $I R$, incidence rate; $a H R$, adjusted hazard ratio; $s H R$, subdistribution hazard ratio; $\mathrm{Cl}$, confidence interval with BS in Taiwan. To the best of our knowledge, no previous large-scale epidemiological investigation has been conducted in the Han Chinese population. The principal findings of our study were (1) that patients with BS did not exhibit a higher risk of IHD compared with the respective risk of the non-BS population; (2) that the 5- and 10-year survival rates of patients with BS were $96.8 \%$ and $95.0 \%$, respectively, which were significantly lower than the rates of sex- and age-matched control subjects; and (3) that male sex was a risk factor of mortality in these patients.

It has been well established that a number of chronic rheumatic disorders, particularly RA and SLE, are associated with substantially increased risk of atherosclerosis and IHD, independent of classical risk factors such as diabetes, hypertension, and dyslipidemia [24, 25]. Accumulating evidence has shown that all stages of atherosclerosis and plaque formation are mainly driven by inflammatory processes [26]. Elevated levels of acute phase reactants, proinflammatory cytokines, and the presence of circulating autoantibodies and specific $\mathrm{T}$ cell subsets observed in RA and SLE lead to a higher atherosclerotic burden [25]. Moreover, a recent randomized double-blind trial revealed that colchicine, an anti-inflammatory medication, reduces the risk of ischemic cardiovascular events among patients who have experienced a recent myocardial infarction, supporting the role of inflammation in the pathogenesis of IHD [27]. 


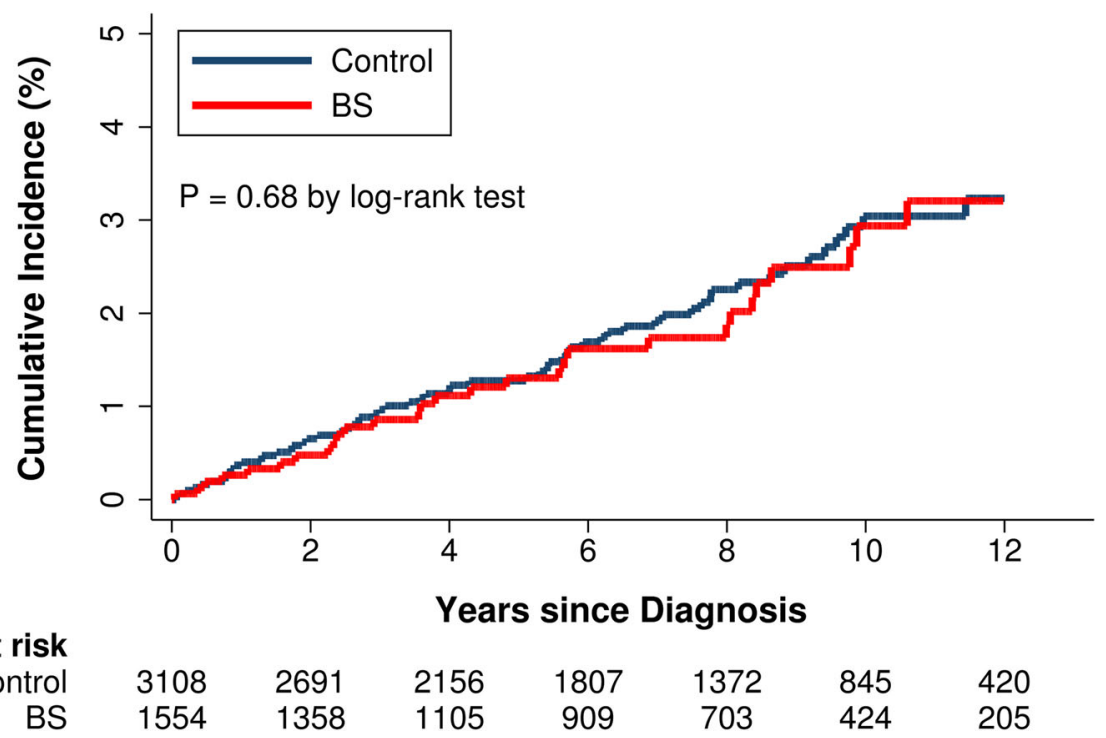

Fig. 2 Cumulative incidence of ischemic heart disease among patients with Behçet's syndrome (BS) and control subjects without BS after propensity score matching

Other factors contributing to IHD in patients with chronic rheumatic diseases include higher prevalence of comorbidities and higher exposure to corticosteroids and NSAIDs. However, not all chronic systemic inflammatory diseases predispose patients to acute coronary events and accelerated atherosclerosis. Some previous studies have demonstrated that the incidence of acute myocardial infarction is not elevated for patients with Crohn's disease or ulcerative colitis $[28,29]$. Moreover, several previous studies have also demonstrated that the mean intima media thickness of carotid arteries is not significantly higher in patients with BS than in healthy controls [14, 30,31]. Our present results indicate that BS does not increase the risk of IHD, as we found a similar risk in the general population. This may be ascribed to the episodic nature of inflammation in BS $[1,3]$, contrary to its relatively persistent and prolonged nature in RA. In addition, the disease activity of BS tends to be attenuated with the passage of time [1,3]. The above may

Table 3 Incidence rate and risk for death in patients with Behçet's syndrome and age-, sex- and index date-matched control subjects

\begin{tabular}{lll}
\hline & $\begin{array}{l}\text { Behçet's syndrome } \\
(\mathbf{n = 1 6 2 5 )}\end{array}$ & $\begin{array}{l}\text { Control } \\
(\mathbf{n}=\mathbf{6 5 0 0})\end{array}$ \\
\hline Death & & \\
Cases, n (\%) & $65(4.0)$ & $157(2.4)$ \\
Person-years & 11,615 & 47,130 \\
IR $(95 \% \mathrm{Cl})^{*}$ & $5.60(4.32-7.13)$ & $3.33(2.83-3.90)$ \\
HR $(95 \% \mathrm{Cl})$ & $1.73(1.30-2.32)$ & Reference \\
\hline
\end{tabular}

"Expressed per 1000 person-years

$H R$, hazard ratio; $I R$, incidence rate; $C l$, confidence interval contribute to the less pronounced cumulative inflammatory vascular damage in BS, as compared to that in RA or SLE. Another explanation for this differential risk of IHD is that the underlying pathophysiological mechanisms of BS are distinct from those of RA/SLE [32]. Thus, it remains to be elucidated whether chronic inflammation is a common critical determinant in the process of accelerated atherosclerosis or disease-specific inflammatory profiles that are more crucial exist.

Recently, a database-driven study in the UK also demonstrated a similar risk of IHD between patients with BS and age- and sex-matched control subjects after adjusting confounding factors in the primary analysis [8]. However, in their sensitivity analysis, which only included incident cases of BS for assessing the primary outcome, the authors reported a significantly higher risk of IHD among patients with BS. The results of this additional analysis regarding the risk of IHD were not consistent with our findings. Nevertheless, the number of incident patients with BS in the UK study was only about 400, which could influence the precision of the estimates, as evidenced by the relatively wider $\mathrm{CI}$ of the HR for the risk of IHD, shown in the sensitivity analysis. Furthermore, lipid-lowering agents were the only medications that were included in their regression model for estimating the risk of IHD. In contrast, in our study, we considered and adjusted the effects of other drugs that may affect the occurrence of IHD, such as corticosteroids, aspirin, and NSAIDs [33]. Additionally, in the UK study, data were extracted from an anonymized primary care database, and authors could not clinically validate the diagnosis of BS, whereas our study was based on a 
A

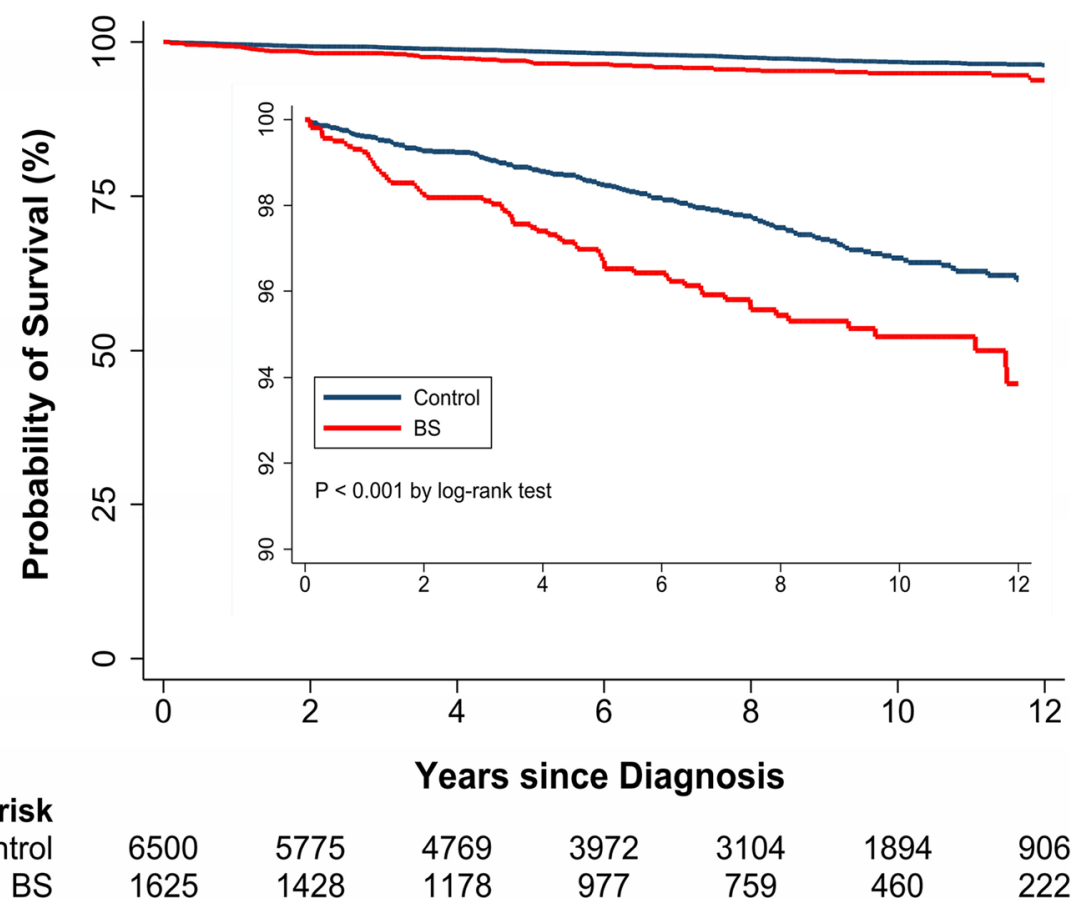

B

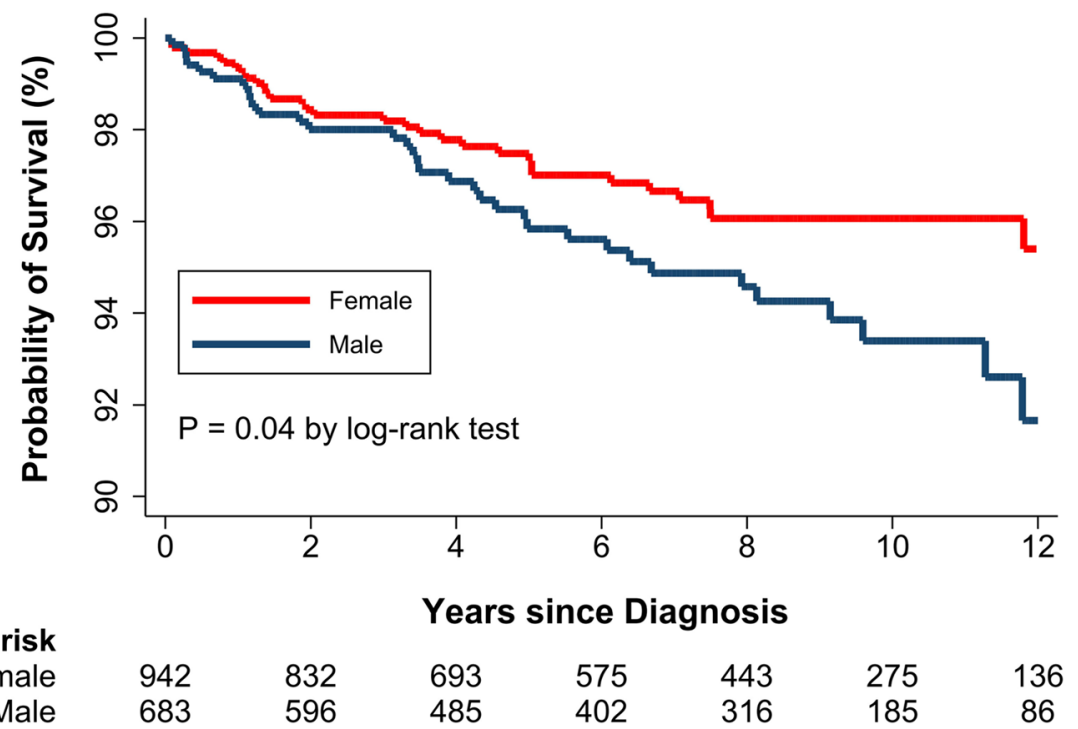

Fig. 3 A Comparison of the survival rate in patients with Behçet's syndrome (BS) and that in age- and sex-matched control subjects. B Survival rate in patients with $\mathrm{BS}$ stratified by sex

nationwide database including patients from general practice, tertiary care, and hospitalized patients. Another explanation for the discrepant results may be that the rate of IHD is higher in Western than Eastern counties [34].
Another study conducted in Korea using a national insurance database and enrolling a large number of patients with BS revealed that the risk of myocardial infarction was $60 \%$ higher than that in the general population [35]. However, similar to the UK study, this 
Korean study also did not address the impact of various medications, which may have detrimental effects on coronary vasculature. The average age of diagnosis of BS was also higher than that in our BS population. Thus, it is possible that the estimated influence of BS on IHD in the Korean study may have been exaggerated, as some confounders were not accounted for.

With respect to long-term prognosis of $\mathrm{BS}$, a recent epidemiological study in the UK showed that the 10-year survival rate is approximately $92 \%$, and male sex carries a higher risk of mortality [8]. A French study enrolling 817 patients with BS revealed that the 3-, 5-, and 10year survival rates were $97.9 \%, 96.7 \%$, and $95.7 \%$, respectively [36]. Another study conducted in Korea using an insurance database estimated that the 5-year survival rate of patients with BS was around 98\% [35]. These findings are comparable to our results.

The strength of our study lies in the use of a nationscale database, enrolling nearly all people living in Taiwan, as well as patients from all levels of health care, to estimate the risk of IHD and mortality. The advantage of this database ensures that selection and referral bias are minimized. In addition, we used propensity score matching and a doubly robust method to account for the confounding effect of age, sex, comorbidities, and various drug usage on the outcome of interest. The diagnostic accuracy of BS in our study was high, as we only included patients with a catastrophic illness certificate. However, there were still some limitations that should be mentioned. First, some important variables regarding lifestyle, such as smoking, were not available in our analysis. However, we extracted information on the percentage of individuals with COPD, both among patients with BS and control subjects, which could be considered as a surrogate marker of smoking, at least to some extent. Second, there was no detailed information on symptoms/signs and laboratory or radiographic results in this claims-database. Thus, we could not evaluate the disease activity status for each patient with BS and analyze its influence on the outcome. Third, we did not have access to the cause of death. Therefore, we could not analyze whether the higher risk of mortality in patients was related to the major vascular manifestations of BS or due to infectious complications. Fourth, although we used validated codes to capture the primary outcome, there could be residual misclassification bias, which was an inherent limitation of the administrative claims database.

\section{Conclusion}

Patients with BS do not exhibit excessive risk of IHD when compared to the respective risk of the non-BS population. It appears that BS cannot be viewed as an independent risk factor of IHD, which is contrary to what is observed for other chronic autoimmune diseases, such as RA or SLE. The 5- and 10-year survival rates among patients with BS were $96.8 \%$ and $95.0 \%$, respectively, which is generally better than the rates of other systemic rheumatic diseases. However, these patients had a 1.7 times higher risk of mortality compared with the general population. Regular and cautious monitoring is still crucial in the management of patients with BS.

\section{Abbreviations \\ BS: Behçet's syndrome; Cl: Confidence interval; COPD: Chronic obstructive lung disease; HR: Hazard ratio; ICD-9-CM: International Classification of Diseases, Ninth Revision, Clinical Modification; IHD: Ischemic heart disease; ISG: International Study Group; NHI: National Health Insurance; NHIR D: National Health Insurance Research Database; NSAIDs: Non-steroidal anti- inflammatory drugs; RA: Rheumatoid arthritis; SLE: Systemic lupus erythematosus}

\section{Acknowledgements}

Not applicable.

\section{Authors' contributions}

CYL is the guarantor of the paper and take responsibility for the integrity of the work as a whole, from its inception to the published article. CYL and $\mathrm{CYH}$ contributed to the study design. All authors contributed to the acquisition of data. CYL contributed to the analysis and interpretation of the data. CYL contributed to the statistical analysis. CYL drafted the manuscript. CYL critically reviewed and edited the manuscript. The authors read and approved the final manuscript.

\section{Funding}

This study was supported by research grants from the National Cheng Kung University Hospital (NCKUH-10903023 and NCKUH-10803032) and the Ministry of Science and Technology (MOST 108-2314-B-006-007-MY2). The funding bodies did not have any role in the design of the study and collection, analysis, and interpretation of data and in writing the manuscript.

\section{Availability of data and materials}

The datasets used and/or analyzed during the current study are available from the corresponding author on reasonable request.

\section{Declarations}

\section{Ethics approval and consent to participate}

The institutional review board of the National Cheng Kung University Hospital approved this study (A-EX-109-017). Informed consent was not required because the datasets were devoid of personally identifiable information.

\section{Consent for publication}

Not applicable.

\section{Competing interests}

The authors declare that they have no competing interests.

\section{Author details}

${ }^{1}$ Division of Rheumatology, Department of Internal Medicine, National Cheng Kung University Hospital, College of Medicine, National Cheng Kung University, No.138, Sheng Li Road, Tainan 704, Taiwan. ${ }^{2}$ Division of Allergy-Immunology-Rheumatology, Department of Internal Medicine, Chi Mei Medical Center, Tainan, Taiwan. ${ }^{3}$ Chia Nan University of Pharmacy and Science, Tainan, Taiwan. ${ }^{4}$ Department of Internal Medicine, National Cheng Kung University Hospital, College of Medicine, National Cheng Kung University, Tainan, Taiwan. ${ }^{5}$ Division of Rheumatology, Allergy, and Immunology, Department of Internal Medicine, Chang Gung Memorial Hospital, Kaohsiung, Taiwan. ${ }^{6}$ College of Medicine, Chang Gung University, Taoyuan, Taiwan. ${ }^{7}$ Institute of Biochemistry, Microbiology and Immunology, Chung Shan Medical University, Taichung, Taiwan. ${ }^{8}$ Division of

Rheumatology, Allergy and Immunology, Department of Internal Medicine, Kaohsiung Chang Gung Memorial Hospital, Chang Gung University College 
of Medicine, No. 123, Ta Pei Road, Niao Sung District, Kaohsiung 83301 Taiwan.

Received: 11 January 2021 Accepted: 21 May 2021

Published online: 04 June 2021

\section{References}

1. Yazici Y, Yurdakul S, Yazici H. Behçet's syndrome. Curr Rheumatol Rep. 2010; 12:429-35.

2. Leonardo NM, McNeil J. Behcet's disease: is there geographical variation? A review far from the silk road. Int J Rheumatol. 2015;2015:945262.

3. Yazici $H$, Seyahi $E$, Hatemi $G$, et al. Behçet syndrome: a contemporary view. Nat Rev Rheumatol. 2018;14:107-19.

4. Yurdakul S, Günaydin I, Tüzün Y, et al. The prevalence of Behçet's syndrome in a rural area in northern Turkey. J Rheumatol. 1988;15:820-2.

5. Shimizu T, Ehrlich GE, Inaba G, et al. Behçet disease (Behçet syndrome). Semin Arthritis Rheum. 1979;8:223-60.

6. Yazici $H$, Chamberlain MA, Tüzün $Y$, et al. A comparative study of the pathergy reaction among Turkish and British patients with Behçet's disease. Ann Rheum Dis. 1984:43:74-5.

7. Kural-Seyahi E, Fresko I, Seyahi N, et al. The long-term mortality and morbidity of Behçet syndrome: a 2-decade outcome survey of 387 patients followed at a dedicated center. Medicine. 2003;82:60-76.

8. Thomas T, Chandan JS, Subramanian A, et al. Epidemiology, morbidity and mortality in Behçet's disease: a cohort study using The Health Improvement Network (THIN). Rheumatology. 2020:59:2785-95.

9. Wolf D, Ley K. Immunity and inflammation in atherosclerosis. Circ Res. 2019; 124:315-27.

10. Bartoloni E, Alunno A, Valentini V, et al. Targeting inflammation to prevent cardiovascular disease in chronic rheumatic diseases: myth or reality? Front Cardiovasc Med. 2018:5:177.

11. Ungprasert P, Suksaranjit P, Spanuchart I, et al. Risk of coronary artery disease in patients with idiopathic inflammatory myopathies: a systematic review and meta-analysis of observational studies. Semin Arthritis Rheum. 2014:44:63-7.

12. Kaklamani VG, Vaiopoulos G, Kaklamanis PG. Behçet's disease. Semin Arthritis Rheum. 1998;27:197-217.

13. Koç Y, Güllü I, Akpek $G$, et al. Vascular involvement in Behçet's disease. J Rheumatol. 1992;19:402-10.

14. Seyahi E, Ugurlu S, Cumali R, et al. Atherosclerosis in Behçet's syndrome. Semin Arthritis Rheum. 2008;38:1-12.

15. Seyahi E, Memisoglu E, Hamuryudan V, et al. Coronary atherosclerosis in Behçet's syndrome: a pilot study using electron-beam computed tomography. Rheumatology. 2004;43:1448-50.

16. Fanouriakis A, Bertsias G, Boumpas DT. Population-based studies in systemic lupus erythematosus: immune thrombocytopenic purpura or 'blooddominant' lupus? Ann Rheum Dis. 2020;79:683-4.

17. Chang SL, Huang YL, Lee MC, et al. Association of varicose veins with incident venous thromboembolism and peripheral artery disease. JAMA. 2018:319:807-17.

18. Criteria for diagnosis of Behçet's disease. International Study Group for Behçet's Disease. Lancet. 1990;335:1078-80,

19. Cheng $\mathrm{CL}$, Lee $\mathrm{CH}$, Chen PS, et al. Validation of acute myocardial infarction cases in the national health insurance research database in Taiwan. J Epidemiol. 2014;24:500-7.

20. Austin PC. Balance diagnostics for comparing the distribution of baseline covariates between treatment groups in propensity-score matched samples. Stat Med. 2009;28:3083-107.

21. Robins JM, Rotnitzky A, Zhao LP. Estimation of regression coefficients when some regressors are not always observed. J Am Stat Assoc. 1994;89:846-66.

22. Bang $H$, Robins JM. Doubly robust estimation in missing data and causal inference models. Biometrics. 2005;61:962-73.

23. Fine JP, Gray RJ. A proportional hazards model for the subdistribution of a competing risk. J Am Stat Assoc. 1999;94:496-509.

24. Nezos A, Evangelopoulos ME, Mavragani CP. Genetic contributors and soluble mediators in prediction of autoimmune comorbidity. J Autoimmun. 2019;104:102317.

25. DeMizio DJ, Geraldino-Pardilla LB. Autoimmunity and inflammation link to cardiovascular disease risk in rheumatoid arthritis. Rheumatol Ther. 2020;7: $19-33$.
26. Matsuura E, Atzeni F, Sarzi-Puttini P, et al. Is atherosclerosis an autoimmune disease? BMC Med. 2014;12:47.

27. Tardif JC, Kouz S, Waters DD, et al. Efficacy and safety of low-dose colchicine after myocardial infarction. N Engl J Med. 2019;381:2497-505.

28. Barnes EL, Beery RM, Schulman AR, et al. Hospitalizations for acute myocardial infarction are decreased among patients with inflammatory bowel disease using a nationwide inpatient database. Inflamm Bowel Dis. 2016;22:2229-37.

29. Osterman MT, Yang YX, Brensinger $C$, et al. No increased risk of myocardial infarction among patients with ulcerative colitis or Crohn's disease. Clin Gastroenterol Hepatol. 2011;9:875-80.

30. Protogerou A, Lekakis J, Stamatelopoulos K, et al. Arterial wall characteristics in patients with Adamantiades-Behçet's disease. Adv Exp Med Biol. 2003; 528:399-404.

31. Caliskan M, Gullu H, Yilmaz S, et al. Cardiovascular prognostic value of vascular involvement in Behcet's disease. Int J Cardiol. 2008;125:428-30.

32. Greco A, De Virgilio A, Ralli M, et al. Behçet's disease: new insights into pathophysiology, clinical features and treatment options. Autoimmun Rev. 2018;17:567-75.

33. Braun J, Baraliakos $X$, Westhoff T. Nonsteroidal anti-inflammatory drugs and cardiovascular risk - a matter of indication. Semin Arthritis Rheum. 2020;50: 285-8.

34. Sasayama S. Heart disease in Asia. Circulation. 2008;118:2669-71.

35. Ahn HS, Lee D, Lee SY, et al. Increased cardiovascular risk and all-cause death in patients with Behçet disease: a Korean nationwide populationbased dynamic cohort study. J Rheumatol. 2020;47:903-8.

36. Saadoun D, Wechsler B, Desseaux K, et al. Mortality in Behçet's disease. Arthritis Rheum. 2010;62:2806-12.

\section{Publisher's Note}

Springer Nature remains neutral with regard to jurisdictional claims in published maps and institutional affiliations.

Ready to submit your research? Choose BMC and benefit from:

- fast, convenient online submission

- thorough peer review by experienced researchers in your field

- rapid publication on acceptance

- support for research data, including large and complex data types

- gold Open Access which fosters wider collaboration and increased citations

- maximum visibility for your research: over $100 \mathrm{M}$ website views per year

At BMC, research is always in progress.

Learn more biomedcentral.com/submissions 